\title{
Formulation of a Galerkin spectral element-Fourier method for three-dimensional incompressible flows in cylindrical geometries
}

\author{
H. M. Blackburn* \\ CSIRO Manufacturing and Infrastructure Technology, \\ PO Box 56, Highett, Vic 3190, Australia \\ S. J. Sherwin \\ Department of Aeronautics, Imperial College London, South Kensington Campus, \\ London, SW7 2AZ, United Kingdom
}

\begin{abstract}
A primitive-variable formulation for simulation of time-dependent incompressible flows in cylindrical coordinates is developed. Spectral elements are used to discretise the meridional semi-plane, coupled with Fourier expansions in azimuth. Unlike previous formulations where special distributions of nodal points have been used in the radial direction, the current work adopts standard Gauss-Lobatto-Legendre nodalbased expansions in both the radial and axial directions. Using a Galerkin projection of the symmetrised cylindrical Navier-Stokes equations all geometric singularities are removed as a consequence of either the Fourier-mode dependence of axial boundary conditions or the shape of the weight function applied in the Galerkin projection. This observation implies that in a numerical implementation, geometrically singular terms can be naively treated by explicitly zeroing their contributions on the axis in integral expressions without recourse to special treatments such as l'Hopital's rule. Exponential convergence of the method both in the meridional semi-plane and in azimuth is demonstrated through application to a three-dimensional analytical solution of the Navier-Stokes equations in which flow crosses the axis.
\end{abstract}

Key words: spectral element, cylindrical coordinates, incompressible flow

* Corresponding author.

Email addresses: hugh.blackburn@csiro.au (H. M. Blackburn), s.sherwin@ic.ac.uk (S. J. Sherwin). 


\section{Introduction}

Spectral element-Fourier discretisations $[1,2,12]$ are well-suited to direct numerical simulation of flows where the geometry exhibits arbitrary complexity in a sectional plane but is infinite or periodic in an orthogonal direction. A number of inter-related simulation technologies have been developed for the spectral element component of these discretisations [6,14]. An obvious extension is to cylindrical coordinate systems, where Fourier expansions can be used in the azimuthal coordinate, while arbitrary complexity can again be accommodated in the meridional semi-plane through use of spectral elements.

Spectral element-based formulations have been proposed for the axisymmetric Stokes problem [7] and the three-dimensional Navier-Stokes system [8,23]. A common feature of these formulations is the use, on elements that touch the cylindrical axis, of Jacobi-polynomial-based expansions in the radial direction. The specialisation of elements that touch the coordinate axis leads (in conforming-element implementations) to undesirable topological constraints on the mesh layout [7]. Besides the obvious specialisation required by the use of a non-standard expansion basis in some elements, these Jacobi polynomials require that some indeterminate axial terms in elliptic equations be evaluated through l'Hopital's rule $[7,23]$, further complicating treatment on the axis.

In the semi-discrete system, the use of Fourier expansions in the azimuthal coordinate brings about a number of requirements on axial boundary conditions $[4,17,23]$. By exploiting these in the weak (Galerkin) form of the elliptic equations in the velocity-correction scheme employed in this paper [13], it can be demonstrated that the use of specialised elements and l'Hopital's rule can both be avoided, and that the resulting method, with Gauss-LobattoLegendre nodal expansions in all elements, preserves exponential convergence in the meridional semi-plane. The method is not specific to the spectral nodal element-Fourier discretisation, or the time-stepping scheme, but is also applicable to other treatments (e.g. finite element-Fourier, $p$-type element-Fourier) that employ Galerkin discretizations in the meridional semi-plane in conjunction with expansion functions that have a natural boundary-interior decomposition. Thus, unlike methods developed in previous work, this formulation demonstrates that the same spectral element or $p$-type finite element treatments commonly used in Cartesian coordinates can be employed in cylindrical coordinates without recourse to basis modification.

Many previous discussions and assessments of cylindrical-coordinate formulations for the incompressible Navier-Stokes system have lacked application to a convenient non-axisymmetric test problem with an analytical solution. Here, we have used the planar Kovasznay flow [15] described in terms of cylindrical coordinates. In this case the problem becomes a three-dimensional, three-com- 
ponent flow, and by appropriate misalignment of the problem and coordinate axes, it can be ensured that all the axial terms in the Navier-Stokes equations are exercised.

In this paper, we give a complete exposition of the formulation, its application within a velocity-correction time-integration scheme, and demonstrate that the method preserves the underlying (here, exponential) convergence properties of the spatial discretization. Further, we demonstrate that the geometrically singular terms arising in the cylindrical description of the Navier-Stokes equations do not contribute to the integral terms of the Galerkin formulation and so can be effectively ignored (or zeroed) in a numerical implementation. While some of the parts of the development can be found elsewhere in the literature, they are not available in a single presentation. To our knowledge, this is the first exposition of a three-dimensional spectral element-Fourier scheme for the Navier-Stokes equations that uniformly employs standard expansion functions in all elements, and for which exponential three-dimensional spatial convergence is demonstrated.

The paper is organised as follows. We start our formulation in $\S 2$ by defining the continuum equations and boundary conditions in cylindrical coordinates. In this section we also introduce the common practice of diagonalising and symmetrising the equations. In $\S 3$ we discuss the numerical discretisation of the Navier-Stokes equations. First we detail the Galerkin formulation and spectral $/ h p$ element discretisation adopted in the current work and illustrate how these components of the formulation remove the effect of the geometric singularities in the problem. We then introduce a temporal discretisation using a velocity-correction scheme which gives rise to a pressure Poisson equation, and show how a naive treatment of the forcing function can compromise convergence. In $\S 4$ we apply the discrete formulation to a non-axisymmetric solution of the Kovasznay problem expressed in terms of cylindrical coordinates and in $\S 5$ we summarise the important steps of the formulation.

\section{Continuum equations and axial boundary conditions}

Starting with the incompressible unsteady Navier-Stokes equations

$$
\begin{aligned}
\partial_{t} \boldsymbol{u}+\boldsymbol{N}(\boldsymbol{u}) & =-\frac{1}{\rho} \nabla p+\nu \nabla^{2} \boldsymbol{u}, \\
\nabla \cdot \boldsymbol{u} & =0
\end{aligned}
$$

and taking the coordinates $z, r, \theta$ to indicate respectively the axial, radial and azimuthal directions in a cylindrical system, we use $\boldsymbol{u}=\boldsymbol{u}(z, r, \theta, t)=$ $(u, v, w)(t)$ to represent the velocity components and $\boldsymbol{N}(\boldsymbol{u})$ to represent non- 
linear advection terms. In addition we have the pressure $p$, density $\rho$ and kinematic viscosity $\nu$. We will take $\boldsymbol{N}(\boldsymbol{u})$ either in their cylindrical-coordinate non-conservative (material-derivative) form

$$
\begin{aligned}
& \boldsymbol{u} \cdot \boldsymbol{\nabla} \boldsymbol{u}=\left(u \partial_{z} u+v \partial_{r} u+\frac{1}{r}\left[w \partial_{\theta} u\right]\right. \\
& u \partial_{z} v+v \partial_{r} v+\frac{1}{r}\left[w \partial_{\theta} v-w w\right], \\
&\left.u \partial_{z} w+v \partial_{r} w+\frac{1}{r}\left[w \partial_{\theta} w+v w\right]\right),
\end{aligned}
$$

or skew-symmetric form

$$
\begin{aligned}
(\boldsymbol{u} \cdot \boldsymbol{\nabla u}+\boldsymbol{\nabla} \cdot \boldsymbol{u u}) / 2= & \left(u \partial_{z} u+v \partial_{r} u+\partial_{z}(u u)+\partial_{r}(v u)\right. \\
& +\frac{1}{r}\left[w \partial_{\theta} u+\partial_{\theta}(w u)+v u\right], \\
& u \partial_{z} v+v \partial_{r} v+\partial_{z}(u v)+\partial_{r}(v v) \\
& +\frac{1}{r}\left[w \partial_{\theta} v+\partial_{\theta}(w v)+v v-2 w w\right], \\
& u \partial_{z} w+v \partial_{r} w+\partial_{z}(u w)+\partial_{r}(v w) \\
& \left.+\frac{1}{r}\left[w \partial_{\theta} w+\partial_{\theta}(w w)+3 v w\right]\right) / 2 .
\end{aligned}
$$

(To consider the above as a Stokes problem, we set $\boldsymbol{N}(\boldsymbol{u})=\mathbf{0}$.)

The velocity must be $2 \pi$-periodic in $\theta$, hence the velocity field can be projected exactly onto a set of two-dimensional complex Fourier modes

$$
\hat{\boldsymbol{u}}_{k}(z, r, t)=\frac{1}{2 \pi} \int_{0}^{2 \pi} \boldsymbol{u}(z, r, \theta, t) \exp (-\mathrm{i} k \theta) \mathrm{d} \theta
$$

where $k$ is an integer wavenumber. The velocity field can be recovered from these complex modes through Fourier series reconstruction

$$
\boldsymbol{u}(z, r, \theta, t)=\sum_{k=-\infty}^{\infty} \hat{\boldsymbol{u}}_{k}(z, r, t) \exp (\mathrm{i} k \theta)
$$

In practice, only a finite number of modes are retained in the calculation, and the conjugate-symmetric property of the Fourier transforms of real variables [5] is exploited, so that the negative- $k$ modes are not required.

The cylindrical-coordinate forms for the gradient and Laplacian of a complex scalar mode are

$$
\nabla_{k}=\left(\partial_{z}(), \partial_{r}(), \frac{\mathrm{i} k}{r}()\right), \quad \nabla_{k}^{2}=\partial_{z}^{2}()+\frac{1}{r} \partial_{r} r \partial_{r}()-\frac{k^{2}}{r^{2}}(),
$$


while for the divergence of a complex vector mode,

$$
\boldsymbol{\nabla} \cdot()_{k}=\partial_{z}()+\frac{1}{r} \partial_{r} r()+\frac{\mathrm{i} k}{r}() .
$$

The components of the Fourier transformed momentum equations (1) can now be written

$$
\begin{aligned}
\partial_{t} \hat{u}_{k}+ & {\left[\boldsymbol{N}(\boldsymbol{u})_{z}\right]_{k}^{\wedge}=} \\
- & \frac{1}{\rho} \partial_{z} \hat{p}_{k}+\nu\left(\partial_{z}^{2}+\frac{1}{r} \partial_{r} r \partial_{r}-\frac{k^{2}}{r^{2}}\right) \hat{u}_{k}, \\
\partial_{t} \hat{v}_{k}+ & {\left[\boldsymbol{N}(\boldsymbol{u})_{r}\right]_{k}^{\wedge}=} \\
- & \frac{1}{\rho} \partial_{r} \hat{p}_{k}+\nu\left(\partial_{z}^{2}+\frac{1}{r} \partial_{r} r \partial_{r}-\frac{k^{2}+1}{r^{2}}\right) \hat{v}_{k}-\nu \frac{2 \mathrm{i} k}{r^{2}} \hat{w}_{k}, \\
\partial_{t} \hat{w}_{k}+ & {\left[\boldsymbol{N}(\boldsymbol{u})_{\theta}\right]_{k}^{\wedge}=} \\
- & \frac{\mathrm{i} k}{\rho r} \hat{p}_{k}+\nu\left(\partial_{z}^{2}+\frac{1}{r} \partial_{r} r \partial_{r}-\frac{k^{2}+1}{r^{2}}\right) \hat{w}_{k}+\nu \frac{2 \mathrm{i} k}{r^{2}} \hat{v}_{k},
\end{aligned}
$$

where $\left[\boldsymbol{N}(\boldsymbol{u})_{z}\right]_{k}$ etc. represent mode- $k$ components of the transformed nonlinear terms.

\subsection{Diagonalization}

Note the coupling of $\hat{v}_{k}$ and $\hat{w}_{k}$ in the viscous terms of equations (14) and (15), which arises through taking the divergence of the viscous stress tensor in cylindrical coordinates. The change of variables

$$
\tilde{v}_{k}=\hat{v}_{k}+\mathrm{i} \hat{w}_{k}, \quad \tilde{w}_{k}=\hat{v}_{k}-\mathrm{i} \hat{w}_{k}
$$

can be introduced to diagonalise these linear terms [19], giving

$$
\begin{aligned}
\partial_{t} \hat{u}_{k}+ & {\left[\boldsymbol{N}(\boldsymbol{u})_{z}\right]_{k}^{\sim}=} \\
- & \frac{1}{\rho} \partial_{z} \hat{p}_{k}+\nu\left(\partial_{z}^{2}+\frac{1}{r} \partial_{r} r \partial_{r}-\frac{k^{2}}{r^{2}}\right) \hat{u}_{k} \\
\partial_{t} \tilde{v}_{k}+ & {\left[\boldsymbol{N}(\boldsymbol{u})_{r}\right]_{k}^{\sim}=} \\
& -\frac{1}{\rho}\left(\partial_{r}-\frac{k}{r}\right) \hat{p}_{k}+\nu\left(\partial_{z}^{2}+\frac{1}{r} \partial_{r} r \partial_{r}-\frac{[k+1]^{2}}{r^{2}}\right) \tilde{v}_{k}, \\
\partial_{t} \tilde{w}_{k}+ & {\left[\boldsymbol{N}(\boldsymbol{u})_{\theta}\right]_{k}^{\sim}=} \\
- & \frac{1}{\rho}\left(\partial_{r}+\frac{k}{r}\right) \hat{p}_{k}+\nu\left(\partial_{z}^{2}+\frac{1}{r} \partial_{r} r \partial_{r}-\frac{[k-1]^{2}}{r^{2}}\right) \tilde{w}_{k}, \\
\partial_{z} \hat{u}_{k}+ & \frac{1}{r} \partial_{r} r \hat{v}_{k}+\frac{\mathrm{i} k}{r} \hat{w}_{k}=0,
\end{aligned}
$$


where also $\left[\boldsymbol{N}(\boldsymbol{u})_{r}\right]_{k} \tilde{k}=\left[\boldsymbol{N}(\boldsymbol{u})_{r}\right]_{\hat{k}}+\mathrm{i}\left[\boldsymbol{N}(\boldsymbol{u})_{\theta}\right]_{\hat{k}}$ and $\left[\boldsymbol{N}(\boldsymbol{u})_{\theta}\right]_{k}=\left[\boldsymbol{N}(\boldsymbol{u})_{r}\right]_{\hat{k}}-$ $\mathrm{i}\left[\boldsymbol{N}(\boldsymbol{u})_{\theta}\right]_{\hat{k}}$. While the equation set contains geometric singularities at $r=0$, we expect on physical grounds that all terms remain finite, since the same equations, expressed in Cartesian coordinates, contain none. Nevertheless, we are concerned with the numerical implications and treatment of these geometric singular terms which can still lead to numerical complications.

\subsection{Symmetrisation}

Following standard practice, the whole set of equations can be symmetrised by premultiplication of equations (17-20) by $r$, leading to

$$
\begin{aligned}
\partial_{t} r \hat{u}_{k} & +r\left[\boldsymbol{N}(\boldsymbol{u})_{z}\right]_{k}^{\sim}= \\
& -\frac{1}{\rho} r \partial_{z} \hat{p}_{k}+\nu\left(\partial_{z} r \partial_{z}+\partial_{r} r \partial_{r}-\frac{k^{2}}{r}\right) \hat{u}_{k}, \\
\partial_{t} r \tilde{v}_{k} & +r\left[\boldsymbol{N}(\boldsymbol{u})_{r}\right]_{k}^{\sim}= \\
& -\frac{1}{\rho}\left(r \partial_{r}-k\right) \hat{p}_{k}+\nu\left(\partial_{z} r \partial_{z}+\partial_{r} r \partial_{r}-\frac{[k+1]^{2}}{r}\right) \tilde{v}_{k}, \\
\partial_{t} r \tilde{w}_{k} & +r\left[\boldsymbol{N}(\boldsymbol{u})_{\theta}\right]_{k}^{\sim}= \\
& -\frac{1}{\rho}\left(r \partial_{r}+k\right) \hat{p}_{k}+\nu\left(\partial_{z} r \partial_{z}+\partial_{r} r \partial_{r}-\frac{[k-1]^{2}}{r}\right) \tilde{w}_{k}, \\
\partial_{z} r \hat{u}_{k} & +\partial_{r} r \hat{v}_{k}+\mathrm{i} k \hat{w}_{k}=0,
\end{aligned}
$$

where the fact that $\partial_{z} r=0$ has been used. Note that as a result of this operation all geometric singularities on the left-hand-sides of this set of equations are removed, while on the right-hand-sides they are, at worst, of type $1 / r$.

\subsection{Axis boundary conditions}

Appropriate conditions to be applied at the axis are derived from solvability requirements and kinematic constraints on scalar and velocity fields at the origin $[3,21,23]$. In principle, regularity conditions at the axis impose constraints on successively higher radial derivatives of the Fourier transformed variables with increasing mode number [24]. However, in practice we only apply the essential boundary conditions to solve the variational equations of the Fourier transformed pressures and velocities, augmented by a minimal subset of radial parity requirements [17]. The required smoothness of the solution is therefore achieved asymptotically as spatial resolution is increased. These considerations 
lead to the following modal dependence of boundary conditions at $r=0$ :

$$
\begin{aligned}
& k=0: \partial_{r} \hat{u}_{0}=\tilde{v}_{0}^{\dagger}=\tilde{w}_{0}=\partial_{r} \hat{p}_{0}=0 \\
& k=1: \hat{u}_{1}^{\dagger}=\tilde{v}_{1}^{\dagger}=\partial_{r} \tilde{w}_{1}=\hat{p}_{1}^{\dagger}=0 \\
& k>1: \hat{u}_{k}^{\dagger}=\tilde{v}_{k}^{\dagger}=\tilde{w}_{k}^{\dagger}=\hat{p}_{k}^{\dagger}=0 .
\end{aligned}
$$

Here, the essential boundary conditions are marked by the superscript $\dagger$ and the remaining terms derive from parity requirements. Note are that it is the non-zero values of $\tilde{w}_{1}$ permitted by its boundary condition that allows flow to cross the axis.

\subsection{Structure of the Fourier-transformed nonlinear terms at the axis}

The axial velocity boundary conditions given by equation (25) allow us to determine the radial structure of the Fourier-transformed nonlinear terms at the axis. We note from the boundary conditions given in equation (25) that all velocity components which have a zero value at the axis must at least have a linear radial decay as they approach the axis. Similarly the two Neumann velocity boundary conditions, $\partial_{r} \hat{u}_{0}=\partial_{r} \tilde{w}_{1}=0$, imply these Fourier velocity variables may have a finite value at the axis. Therefore we note that the only possible non-zero velocity modes at the axis are $\hat{u}_{0}, \hat{v}_{1}=\tilde{w}_{1} / 2$ and $\hat{w}_{1}=\mathrm{i} \tilde{w}_{1} / 2$ where we have applied the inverse of relationship (16) to get the last two conditions.

Considering the velocity components which may be finite at the axis, we proceed by noting that through the Fourier convolution theorem (see e.g. [5]),

$$
\hat{c}_{k}=\widehat{a b}_{k}=\sum_{p+q=k} \hat{a}_{p} \hat{b}_{q}, \quad k, p, q \in \mathbb{I}
$$

only modes $k=0, k=1$ and $k=2$ (and negative- $k$ conjugates) of any quadratic nonlinear terms, equations (3-5) and (6-8), can have non-zero values at $r=0$. Explicitly we note that for $k=0$ we have $(p, q)=(0,0),(-1,1)$ and $(1,-1)$; for $k=1:(p, q)=(0,1)$ and $(1,0)$; and for $k=2:(p, q)=(1,1)$.

In determining the radial behaviour of the nonlinear terms at the axis we shall first consider the geometrically singular terms arising in equations (35 ) and (6-8) as a consequence of the $1 / r$ factors. We will then discuss the contributions of the remaining terms. The first observation we can make is that all the bracketed nonlinear terms premultiplied by $1 / r$ in equations $(3-5)$ evaluate to zero at $r=0$. This is to be expected, since we know that even when multiplied by $1 / r$, they must remain finite. The point can be demonstrated by noting that either the terms are individually zero as a direct consequence 
of the boundary conditions (25), or a summation of terms exactly cancel. For example when $r=0$ a contribution to the $k=2$ Fourier-transformed squarebracketed term in equation (4) will arise from $\hat{w}_{1} \partial_{\theta} \hat{v}_{1}-\hat{w}_{1} \hat{w}_{1}$, which could potentially have a finite axial contribution. However upon insertion of the relationship that $\hat{v}_{1}=\tilde{w}_{1} / 2$ and $\hat{w}_{1}=\mathrm{i} \tilde{w}_{1} / 2$ and using Fourier differentiation with respect to $\theta$ it will be found that $\hat{w}_{1} \partial_{\theta} \hat{v}_{1}-\hat{w}_{1} \hat{w}_{1}$ sums to zero.

Even though the square-bracketed terms in equations (3-5) evaluate or sum to zero, we still need to know the radial decay of these terms as they approach the axis, since when multiplied by the $1 / r$ factor the product may still have a finite limit. We therefore examine how these Fourier-transformed (bracketed) terms approach zero as as function of $r$. The three possibilities after application of boundary conditions (25) and using the convolution and differentiation theorems are: $(i)$ linear decay, as the product of zero and a finite term; (ii) quadratic decay, either directly as the product of two zero terms or the difference of (asymptotically) equal linear terms; (iii) quartic decay, as the difference of equal quadratic terms. Of these, only the linear terms will, when premultiplied by $1 / r$, have a finite analytical limit at the axis which could be determined (if required) using l'Hopital's rule. The outcome of the analysis for the square-bracketed terms is as follows:

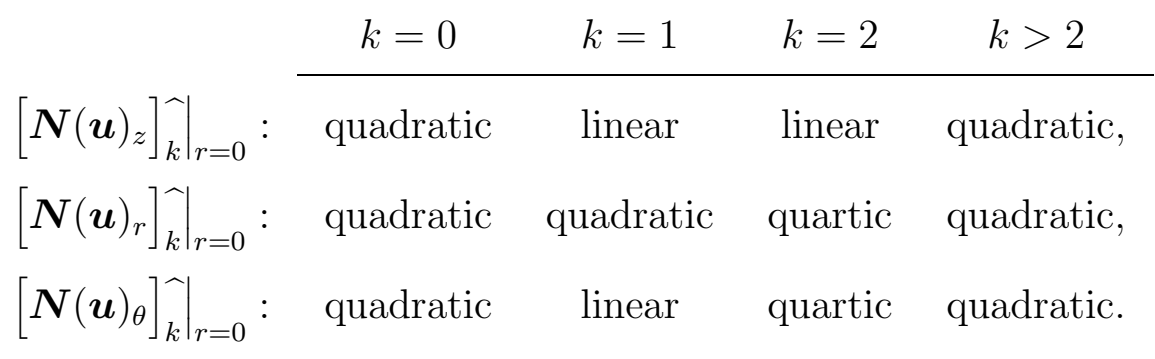

Note that for $k=0$, none of the terms in question is (when premultiplied by $1 / r)$ non-zero at $r=0$.

Finally we consider the axial form of the remaining terms of the Fouriertransformed nonlinear terms. We will only consider the non-conservative forms (3-5), but the conclusions for the radial structure of the skew-symmetric forms are the same. By considering which Fourier modes contribute to the Fouriertransformed nonlinear terms and using the fact that only $\hat{u}_{0}$ and $\tilde{w}_{1}$ can be nonzero at the axis we obtain that the limit of the Fourier-transformed nonlinear 
terms are:

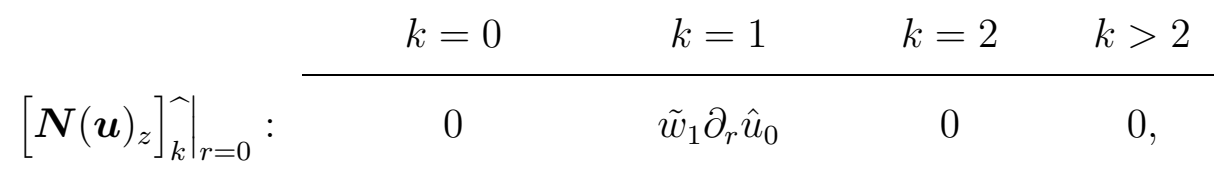

$$
\begin{aligned}
& {\left.\left[\boldsymbol{N}(\boldsymbol{u})_{r}\right]_{k}\right|_{r=0}: \operatorname{Re}\left(\tilde{w}_{1} \partial_{r} \tilde{w}_{1}\right) / 2 \quad \hat{u}_{0} \partial_{z} \tilde{w}_{1} / 2 \quad \tilde{w}_{1} \partial_{r} \tilde{w}_{1} / 4 \quad 0,} \\
& {\left.\left[\boldsymbol{N}(\boldsymbol{u})_{\theta}\right]_{k}\right|_{r=0}: \quad \quad 0 \quad \mathrm{i} \hat{u}_{0} \partial_{z} \tilde{w}_{1} / 2 \quad \mathrm{i} \tilde{w}_{1} \partial_{r} \tilde{w}_{1} / 4 \quad 0 .}
\end{aligned}
$$

For what follows, the most important feature in the above result is that for $k=0$, the radial component of the nonlinear terms can be non-zero at $r=0$ as a consequence of the non-linear contribution from the coupled-velocitycomponent variable $\tilde{w}_{1}$ associated with cross-axial flow.

\section{Discretisation}

\subsection{Galerkin treatment of elliptic operators}

After the symmetrisation, all elliptic scalar operators in the (Navier-)Stokes problem are of type

$$
\partial_{z} r \partial_{z} \hat{c}_{k}+\partial_{r} r \partial_{r} \hat{c}_{k}-\frac{\sigma^{2}}{r} \hat{c}_{k}=r \hat{f}_{k}
$$

where $\hat{c}_{k}, \hat{f}_{k}$, are complex scalar variables and $\sigma^{2}$ is a Fourier-mode constant, e.g. $\sigma^{2}=(k+1)^{2}$ in equation (22). These equations are converted to their weak form in conjunction with a projection $[11,22]$; they are multiplied by a weight function $\phi$ (defined to be zero on all Dirichlet boundaries), and integrated by parts over the solution domain $\Omega$, boundary $\Gamma=\Gamma_{\mathrm{D}}+\Gamma_{\mathrm{N}}$ :

$$
\int_{\Omega} r \partial_{z} \phi \partial_{z} \hat{c}_{k}+r \partial_{r} \phi \partial_{r} \hat{c}_{k}+\frac{\sigma^{2}}{r} \phi \hat{c}_{k} \mathrm{~d} \Omega=-\int_{\Omega} r \phi \hat{f}_{k} \mathrm{~d} \Omega+\int_{\Gamma_{\mathrm{N}}} r \phi h \mathrm{~d} \Gamma,
$$

where $h$ represent non-essential (e.g. Neumann, $\partial_{n} \hat{c}_{k}$ ) boundary conditions on boundary partition $\Gamma_{\mathrm{N}}$.

We impose all Dirichlet boundary conditions using a strong enforcement and maintaining the symmetry of the problem. Mathematically this requires that the Dirichlet boundary conditions are 'lifted' into the interior of the domain by some known function. This known lifted solution then modifies the right-handside of the original problem leaving a problem with homogeneous boundary conditions to be solved. In practice the numerical solution is decomposed into a known, Dirichlet-boundary-condition-satisfying contribution and an unknown 


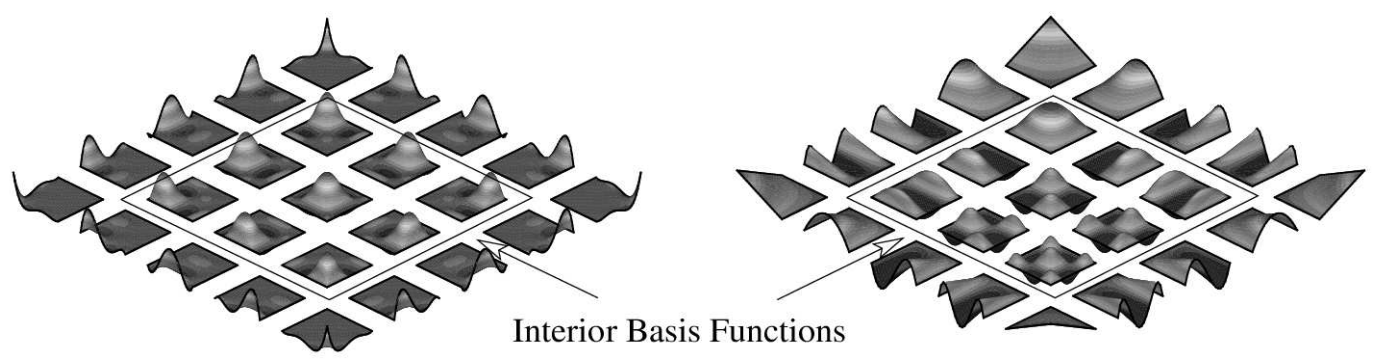

Fig. 1. Shape of expansion bases for a 4th order polynomial expansion using nodal spectral elements (left) and $p$-type hierarchical expansion (right). Note that in both cases the basis functions can be decomposed into boundary contributions which have support on the boundary and interior contributions which are identically zero on the boundary.

contribution with homogeneous Dirichlet boundary conditions. This is always possible since the elliptic operator is linear $[11,14]$. The argument that follows is not necessarily true for weak enforcement of the Dirichlet boundary conditions, e.g. through a penalty method.

We note that for cases where zero-Neumann conditions are imposed at the axis, the Fourier constants $\sigma^{2}=0$ and thus the potentially singular terms in operators of the form (29) do not need further consideration.

Clearly, in order to reduce the $\sigma^{2} / r$ singularities when $\sigma^{2} \neq 0$ at least one of $\hat{c}_{k}, \phi$ in the third term of equation (29) must be zero at $r=0$, while the other may be either zero or remain finite. If the numerator of the term $\sigma^{2} \phi \hat{c}_{k} / r$ contains one factor of $r$ then the finite limit of this expression can be evaluated using l'Hopital's rule. If both $\hat{c}_{k}$ and $\phi$ are zero at $r=0$, then evaluation using l'Hopital's rule is not required, since the numerator of $\sigma^{2} \phi \hat{c}_{k} / r$ will (at worst) contain a $r^{2}$ factor and so the quotient will necessarily be zero at $r=0$. By inspection of equations (21-23) and (25) it can be seen that for all cases when the Fourier constant $\sigma^{2} \neq 0$, we impose the Dirichlet boundary condition $\hat{c}_{k}=0$ at the axis. Further, due to the strong enforcement of Dirichlet boundary conditions in the Galerkin formulation, $\phi(r=0)=0$ also. This simple observation is the key to the present formulation. The practical implication of this observation is that even if we independently construct geometrically singular terms of the form $\hat{c}_{k} / r$, we do not need to evaluate the indeterminate limit (for example using l'Hopital's rule) since in the Galerkin formulation they are only required as a product of the form $\sigma^{2} \phi \hat{c}_{k} / r$. Therefore we are free to naively set the indeterminate limit of $\hat{c}_{k} / r$ to zero (or any non-infinite value) without loss of generality in the Galerkin approximation. 


\subsection{Expansion bases}

We now need to discuss the form of the expansion bases for $\hat{c}_{k}, \phi$. Ideally, expansion bases are required which are zero at the axis for some variables and modes, but which also have support along the axis whenever $\sigma^{2}=0$. If global (e.g. Chebychev, Legendre) expansions are employed, then special combinations of the underlying expansions can be made in order to ensure the functions satisfy these conditions, mode-by-mode and variable-by-variable [24]. On the other hand, sets of expansion bases that allow a boundary-interior decomposition - sets which have interior basis functions with zero support on the boundary and boundary basis functions that have support on the boundary of the region (see Fig. 1) - automatically satisfy this requirement. For these expansion sets, basis functions that are non-zero at the axis can be partitioned out of the problem as required, and the remaining functions will satisfy the homogeneous essential boundary conditions.

Typical bases designed for $C_{0}$ continuity meet the requirements; suitable candidates are provided by $h$ - and $p$-type finite elements [18], nodal- and hierarchical spectral element expansions [6,14] and B-splines [16]. The present implementation has employed standard, Gauss-Lobatto-Legendre-nodal-based spectral elements, but any of the above alternatives could have been chosen. The partitioning of basis functions in order to strongly satisfy essential boundary conditions is standard practice in finite element and spectral element methodology.

\subsection{Temporal discretisation}

The Galerkin formulation using the boundary-interior decomposed basis has been sufficient to remove the geometric singularities in the symmetrised form of the governing equations. In principle, any Navier-Stokes time discretisation scheme based on this primitive formulation will therefore correctly deal with the geometric singularities arising as a consequence of the cylindrical coordinate system. Nevertheless many commonly used discretisations, including the one adopted in this paper, employ a pressure Poisson equation. Since this equation is constructed by taking the divergence of the momentum equation (1), it is possible for geometrically singular terms to contribute to the domain-integral forcing term in the Galerkin projection of this equation. Before elaborating on this point in $\S 3.4$, we first outline the temporal discretisation adopted in this paper. 


\subsubsection{Velocity-correction time integration scheme}

The temporal discretisation used is a projection scheme, based on backwards differencing in time. As originally described [13], this was characterised as an operator-splitting scheme, but more recently [10] it has been shown that the method is one of a class of velocity-correction projection schemes, which is how it will be referred to here. For completeness, and as a guide to the cylindricalcoordinate treatment to follow, the scheme will be briefly presented in its semi-discrete form.

The projection scheme requires the solution of a pressure Poisson equation to (approximately) maintain solenoidality of the velocity, a point that was not directly addressed in $\S 2$. The treatment of this elliptic operator at $r=0$ carries with it the same considerations as outlined in $\S 3.1$ (the Fourier mode constant and the axial boundary conditions for $\hat{p}_{k}$ are the same as for $\hat{u}_{k}$ ), however it will transpire in $\S \S 3.3 .2,3.4$ that careful examination is required of the domain- and boundary-integral forcing terms in this equation.

Backwards time differencing is used to approximate a derivative at the new time level $(n+1)$ through

$$
\partial_{t}()^{(n+1)}=\frac{1}{\Delta t} \sum_{q=0}^{J} \alpha_{q}()^{(n-q+1)}+O(\Delta t)^{J+1},
$$

and in addition, a method is needed to explicitly extrapolate previous terms to the new time level, which is achieved through polynomial approximation

$$
()^{(n+1)}=\sum_{q=0}^{J-1} \beta_{q}()^{(n-q)}+O(\Delta t)^{J} .
$$

The discrete weights $\alpha_{q}, \beta_{q}$, for schemes of order up to $J=3$ appear e.g. in $[13,14]$.

The time-step for the velocity-correction scheme commences with solution of a pressure Poisson equation, followed by a pressure-gradient update

$$
\begin{aligned}
r \boldsymbol{u}^{*} & =-\sum_{q=1}^{J} \alpha_{q} r \boldsymbol{u}^{(n-q)}-\Delta t \sum_{q=0}^{J-1} \beta_{q} r \boldsymbol{N}\left(\boldsymbol{u}^{(n-q)}\right) \\
r \nabla^{2} p^{(n+1)} & =\frac{\rho}{\Delta t} r \boldsymbol{\nabla} \cdot \boldsymbol{u}^{*}, \quad \text { with } \\
r \partial_{n} p^{(n+1)} & =-r \rho \boldsymbol{n} \cdot \sum_{q=0}^{J-1} \beta_{q}\left(\boldsymbol{N}\left(\boldsymbol{u}^{(n-q)}\right)+\nu \boldsymbol{\nabla} \times \boldsymbol{\nabla} \times \boldsymbol{u}^{(n-q)}+\partial_{t} \boldsymbol{u}^{(n-q)}\right) \\
r \boldsymbol{u}^{* *} & =r \boldsymbol{u}^{*}-\frac{\Delta t}{\rho} r \boldsymbol{\nabla} p^{(n+1)}
\end{aligned}
$$


where $\boldsymbol{n}$ is the domain unit outward normal, and equation (34) is used to estimate a Neumann pressure boundary condition on (e.g. far-field, solid) boundaries where no other condition is explicitly set. The enforcement of the solenoidality of $\nabla^{2} \boldsymbol{u}^{(n-q)}=\boldsymbol{\nabla} \boldsymbol{\nabla} \cdot \boldsymbol{u}^{(n-q)}-\boldsymbol{\nabla} \times \boldsymbol{\nabla} \times \boldsymbol{u}^{(n-q)}$ in forming equation (34) is essential to the time-accuracy of the scheme [13]. The step is completed by applying a viscous correction through solution of a Helmholtz (elliptic) equation for $\boldsymbol{u}^{(n+1)}$

$$
r \nabla^{2} \boldsymbol{u}^{(n+1)}-\frac{r \alpha_{0}}{\nu \Delta t} \boldsymbol{u}^{(n+1)}=-\frac{r \boldsymbol{u}^{* *}}{\nu \Delta t}
$$

(actually, a set of scalar Helmholtz equations) together with appropriate velocity boundary conditions at time $(n+1) \Delta t$.

When it comes to applying this scheme to equations (21-24), the first substep, equations (32-35), may conveniently be applied to the primitive variables $\left(\hat{u}_{k}\right.$, $\hat{v}_{k}, \hat{w}_{k}, \hat{p}_{k}$ ), while the second substep, equation (36), may be applied to the diagonalising variables $\left(\hat{u}_{k}, \tilde{v}_{k}, \tilde{w}_{k}, \hat{p}_{k}\right)$.

The use of higher derivatives in computing the rotational forms used in the pressure boundary conditions implies the use of a high-order spatial discretisation in the algorithm of [13], but other forms of the velocity-correction schemes do not carry this restriction, as pointed out in [10]. We will not pursue this issue further here, and will employ a high-order spatial discretisation, but note that this does not imply a limitation on our central conclusions.

\subsubsection{Solution algorithm}

We now write the full solution algorithm for a single timestep, and defer further discussion of axial singularities to $\S 3.4$.

The component form for the pressure Poisson equation (33), exploiting the fact that $\partial_{z} r=0$, is

$$
\left(\partial_{z} r \partial_{z}+\partial_{r} r \partial_{r}-\frac{k^{2}}{r}\right) \hat{p}^{(n+1)}=\frac{\rho}{\Delta t}\left(\partial_{z} r \hat{u}_{k}^{*}+\partial_{r} r \hat{v}_{k}^{*}+\mathrm{i} k \hat{w}_{k}^{*}\right)
$$

where each component appearing in the right-hand-side divergence is

$$
\begin{aligned}
& r \hat{u}_{k}^{*}=-\sum_{q=1}^{J} \alpha_{q} r \hat{u}^{(n-q)}-\Delta t \sum_{q=0}^{J-1} \beta_{q} r\left[\boldsymbol{N}\left(\boldsymbol{u}^{(n-q)}\right)_{z}\right]_{k}, \\
& r \hat{v}_{k}^{*}=-\sum_{q=1}^{J} \alpha_{q} r \hat{v}^{(n-q)}-\Delta t \sum_{q=0}^{J-1} \beta_{q} r\left[\boldsymbol{N}\left(\boldsymbol{u}^{(n-q)}\right)_{r}\right]_{k}^{\wedge}, \\
& \hat{w}_{k}^{*}=-\sum_{q=1}^{J} \alpha_{q} \hat{w}^{(n-q)}-\Delta t \sum_{q=0}^{J-1} \beta_{q}\left[\boldsymbol{N}\left(\boldsymbol{u}^{(n-q)}\right)_{\theta}\right]_{k}^{\wedge}
\end{aligned}
$$


Note that since $\partial_{r} r \hat{v}_{k}^{*}=r \partial_{r} \hat{v}_{k}^{*}+\hat{v}_{k}^{*}$, there are components in the second and third terms of the right-hand-side of equation (37) that do not directly contain a factor of $r$. Equation (37) is augmented with the boundary conditions

$$
\begin{aligned}
r \partial_{n} \hat{p}^{(n+1)}=- & r \rho \sum_{q=0}^{J-1} \\
& n_{z}\left[\beta_{q}\left(\boldsymbol{N}\left(\boldsymbol{u}^{(n-q)}\right)+\nu \boldsymbol{\nabla} \times \boldsymbol{\nabla} \times \boldsymbol{u}^{(n-q)}+\partial_{t} \boldsymbol{u}^{(n-q)}\right)_{z}\right]_{k}, \\
& +n_{r}\left[\beta_{q}\left(\boldsymbol{N}\left(\boldsymbol{u}^{(n-q)}\right)+\nu \boldsymbol{\nabla} \times \boldsymbol{\nabla} \times \boldsymbol{u}^{(n-q)}+\partial_{t} \boldsymbol{u}^{(n-q)}\right)_{r}\right]_{k},
\end{aligned}
$$

on the non-axial segment of $\Gamma_{\mathrm{N}}$ for $\hat{p}_{k}$, and solved using the Galerkin formulation outlined in $\S 3.1$. For $\hat{p}_{0}$ we have $\partial_{r} \hat{p}_{0}=0$ on the axial segment of $\Gamma_{\mathrm{N}}$, while the axis is a location of $\hat{p}_{k}=0$ essential boundary conditions for all other modes. Note that terms multiplying $n_{\theta}$ are not required as the domain is axisymmetric. The real and imaginary contributions to the mode- $k$ rotational boundary terms are, with $\hat{\zeta}_{k}=\partial_{z} \hat{v}_{k}-\partial_{r} \hat{u}_{k}$,

$$
\begin{aligned}
& \operatorname{Re}\left(r\left[(\boldsymbol{\nabla} \times \boldsymbol{\nabla} \times \boldsymbol{u})_{z}\right]_{k}^{\uparrow}\right)=\left(1+r \partial_{r}\right) \operatorname{Re}\left(\hat{\zeta}_{k}\right)-k \partial_{z} \operatorname{Im}\left(\hat{w}_{k}\right)+\frac{k^{2}}{r} \operatorname{Re}\left(\hat{u}_{k}\right), \\
& \operatorname{Im}\left(r\left[(\boldsymbol{\nabla} \times \boldsymbol{\nabla} \times \boldsymbol{u})_{z}\right]_{k}^{\uparrow}\right)=\left(1+r \partial_{r}\right) \operatorname{Im}\left(\hat{\zeta}_{k}\right)+k \partial_{z} \operatorname{Re}\left(\hat{w}_{k}\right)+\frac{k^{2}}{r} \operatorname{Im}\left(\hat{u}_{k}\right), \\
& \operatorname{Re}\left(r\left[(\boldsymbol{\nabla} \times \boldsymbol{\nabla} \times \boldsymbol{u})_{r}\right]_{k}^{\uparrow}\right)=-r \partial_{z} \operatorname{Re}\left(\hat{\zeta}_{k}\right)-\left(\frac{k}{r}+k \partial_{r}\right) \operatorname{Im}\left(\hat{w}_{k}\right)+\frac{k^{2}}{r} \operatorname{Re}\left(\hat{v}_{k}\right), \\
& \operatorname{Im}\left(r\left[(\boldsymbol{\nabla} \times \boldsymbol{\nabla} \times \boldsymbol{u})_{r}\right]_{k}^{\uparrow}\right)=-r \partial_{z} \operatorname{Im}\left(\hat{\zeta}_{k}\right)+\left(\frac{k}{r}+k \partial_{r}\right) \operatorname{Re}\left(\hat{w}_{k}\right)+\frac{k^{2}}{r} \operatorname{Im}\left(\hat{v}_{k}\right) .
\end{aligned}
$$

To complete the projection step we initially determine $r \boldsymbol{u}^{* *}$ as

$$
\begin{aligned}
r \hat{u}_{k}^{* *} & =r \hat{u}_{k}^{*}-\Delta t r \partial_{z} \hat{p}_{k}^{(n+1)} \\
r \hat{v}_{k}^{* *} & =r \hat{v}_{k}^{*}-\Delta t r \partial_{r} \hat{p}_{k}^{(n+1)}, \\
r \hat{w}_{k}^{* *} & =r \hat{w}_{k}^{*}-\Delta t i k \hat{p}_{k}^{(n+1)},
\end{aligned}
$$

where we note the factor of $r$ now applied to $\hat{w}_{k}^{*}$ in (48). The correction step is constructed by applying the change of variables required to diagonalise the viscous terms, i.e.

$$
r \tilde{v}_{k}^{* *}=r \hat{v}_{k}^{* *}+\mathrm{i} r \hat{w}_{k}^{* *}, \quad r \tilde{w}_{k}^{* *}=r \hat{v}_{k}^{* *}-\mathrm{i} r \hat{w}_{k}^{* *},
$$


and the Helmholtz equation for each velocity component then becomes

$$
\begin{aligned}
& \left(\partial_{z} r \partial_{z}+\partial_{r} r \partial_{r}-\frac{k^{2}}{r}-\frac{r \alpha_{0}}{\nu \Delta t}\right) \hat{u}_{k}^{(n+1)}=-\frac{r}{\nu \Delta t} \hat{u}_{k}^{* *}, \\
& \left(\partial_{z} r \partial_{z}+\partial_{r} r \partial_{r}-\frac{(k+1)^{2}}{r}-\frac{r \alpha_{0}}{\nu \Delta t}\right) \tilde{v}_{k}^{(n+1)}=-\frac{r}{\nu \Delta t} \tilde{v}_{k}^{* *}, \\
& \left(\partial_{z} r \partial_{z}+\partial_{r} r \partial_{r}-\frac{(k-1)^{2}}{r}-\frac{r \alpha_{0}}{\nu \Delta t}\right) \tilde{w}_{k}^{(n+1)}=-\frac{r}{\nu \Delta t} \tilde{w}_{k}^{* *},
\end{aligned}
$$

which are solved by Galerkin projection. Finally, the Fourier-transformed radial and azimuthal velocities

$$
\hat{v}_{k}^{(n+1)}=\left(\tilde{v}_{k}^{(n+1)}+\tilde{w}_{k}^{(n+1)}\right) / 2, \quad \hat{w}_{k}^{(n+1)}=\mathrm{i}\left(\tilde{w}_{k}^{(n+1)}-\tilde{v}_{k}^{(n+1)}\right) / 2,
$$

are recomputed to complete the time step.

In the Helmholtz equations (50-52), we now have an extra term on the lefthand-side, compared to the canonical form (28), but the treatment of the axial terms on the left-hand-sides of the equations is not altered and all the previous conclusions regarding geometrically singular terms still hold. The Galerkin projections of (50-52) are of the form

$$
\int_{\Omega} r \partial_{z} \phi \partial_{z} \hat{c}_{k}+r \partial_{r} \phi \partial_{r} \hat{c}_{k}+\frac{\sigma^{2}}{r} \phi \hat{c}_{k}+r \gamma^{2} \phi \hat{c}_{k} \mathrm{~d} \Omega=-\int_{\Omega} r \phi \hat{f}_{k} \mathrm{~d} \Omega+\int_{\Gamma_{\mathrm{N}}} r \phi h \mathrm{~d} \Gamma,
$$

where $\gamma^{2}=\alpha_{0} / \nu t$ is a Helmholtz constant multiplying the new term.

\subsection{Geometrically singular terms in the pressure Poisson equation}

To recap, we recall that all geometrically singular terms arising in the Helmholtz equations (50-52) do not contribute to the integrals in the weak Galerkin projection of the discrete problems. The right-hand-sides of these equations are all multiplied by a factor of $r$, which zeros any contribution made by the indeterminate, but finite, parts of the geometrically singular non-linear terms (§ 2.4) implicit in the definition of $\tilde{\boldsymbol{u}}_{k}^{* *}$. The left-hand-side terms do not contribute through a combination of factors: firstly, for certain terms where there are Neumann boundary conditions on the axis, the Fourier-mode constant, $\sigma^{2}$, is zero. Secondly, the strong imposition of Dirichlet boundary conditions (given in 25) means that both the variable and the weight function are zero at the axis, which in turn implies that the numerators of the singular expressions approach zero faster than the denominators.

Now if we consider the pressure Poisson equation (37), a similar argument holds for the left-hand-side geometrically singular terms as we above applied to the Helmholtz equations. However the right-hand side of equation (37) is 
of a slightly different form to those for the Helmholtz equations, owing to the divergence operator involved in evaluating this term (see equation (33)). To appreciate this point we can rewrite the right-hand side of equation (37) as

$$
\frac{\rho}{\Delta t}\left(\partial_{z} r \hat{u}_{k}^{*}+\partial_{r} r \hat{v}_{k}^{*}+\mathrm{i} k \hat{w}_{k}^{*}\right)=\frac{\rho}{\Delta t}\left(\partial_{z} r \hat{u}_{k}^{*}+r \partial_{r} \hat{v}_{k}^{*}+\hat{v}_{k}^{*}+\mathrm{i} k \hat{w}_{k}^{*}\right) .
$$

The intermediate velocity field $\hat{\boldsymbol{u}}_{k}^{*}$ is constructed as sum of factors of the (past) velocities and nonlinear terms, as shown in equations (32) and (38-40). We know from the analysis of $\S 2.4$ that there are indeterminate, but finite, nonlinear terms at the axis for $k=1,2$. However, for these pressure modes, our strong enforcement of the zero-Dirichlet axial boundary condition means that $\phi(r=0)=0$, hence those indeterminate forms do not require evaluation in the Galerkin projection. For $k=0$, all the axial contributions to $\hat{v}_{0}^{*}$ are determinate and explicitly computable. We shall discuss other (incorrect) ways of dealing with these pressure forcing terms in $\S 5$.

Having dealt with the area-integral forcing terms on the right of equation (37), we finally consider the geometrically singular terms of $\boldsymbol{\nabla} \times \boldsymbol{\nabla} \times \boldsymbol{u}$ in equations (42-45). Obviously, these only need attention for $k>0$, and they can only make a contribution where a non-axial segment of $\Gamma_{N}$ touches the axis, since along the axis itself, the pressure boundary conditions are supplied. Again, all the terms are indeterminate and finite, rather than singular: $\hat{u}_{k}=0$ at the axis, $k>0$, which accounts for the geometrically singular terms in $(42,43), \hat{v}_{k}=0$, $\hat{w}_{k}=0$ at the axis, $k>1$, while at $k=1$ the geometrically singular terms in $(44,45)$ evaluate to the real and imaginary parts of $\tilde{v}_{1}$, which are both zero at the axis. Thus for $k>0$, all the $1 / r$-premultiplied terms are indeterminate but finite, however, again the pressure test function $\phi(r=0)=0$ in these cases, meaning their evaluation is never required.

In summary, all the geometrically singular terms in the elliptic equations either evaluate to zero, or are finite but never require evaluation as a consequence of the Galerkin projection formulation and the modal structure of the axis boundary conditions.

\section{Test case}

The test case we have considered is the Kovasznay flow [15], an analytical twodimensional steady solution to the incompressible Navier-Stokes equations. In Cartesian $(x, y)$ coordinates, this is defined as

$$
\begin{aligned}
& u=1-\exp (\lambda x) \cos (2 \pi y), \\
& v=(2 \pi)^{-1} \lambda \exp (\lambda x) \sin (2 \pi y), \\
& p=(1-\exp \lambda x) / 2,
\end{aligned}
$$




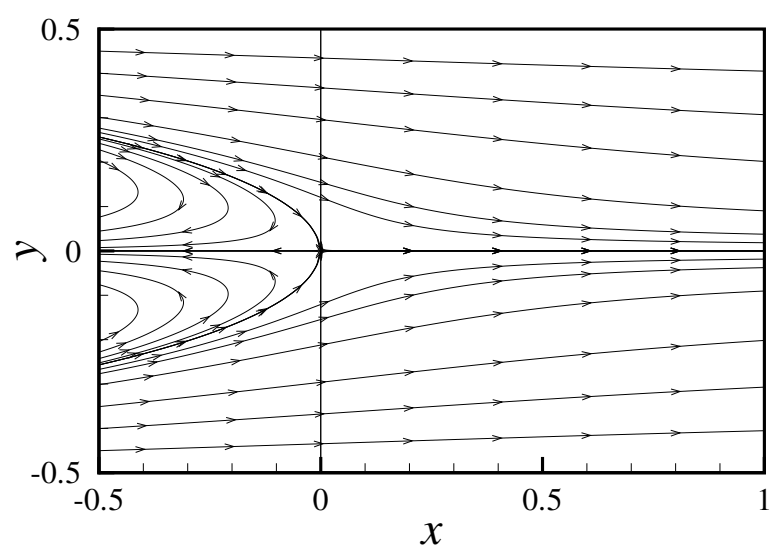

Fig. 2. Streamlines of the Kovasznay flow for $R e=40$, illustrated in Cartesian coordinates.

where $\lambda=R e / 2-\left(R e^{2} / 4+4 \pi^{2}\right)^{1 / 2}, R e \equiv 1 / \nu$. While this is a steady flow, we seek only to examine the spatial convergence properties of the numerical scheme, as temporal convergence is already established [13]. Streamlines of the flow, for $R e=40$, are shown in Fig. 2 .

In cylindrical coordinates (with $z$ now labelling the axial direction, $r$ the radial direction and $\theta$ the azimuthal), and allowing an arbitrary rotation $\Theta$ of the solution coordinates about the Cartesian $x$ axis and an arbitrary displacement $-\Delta$ of the Cartesian $x$-axis from the cylindrical $z$-axis, this becomes

$$
\begin{aligned}
u & =1-\exp (\lambda z) \cos (2 \pi[r \cos (\theta+\Theta)+\Delta]), \\
v & =(2 \pi)^{-1} \lambda \exp (\lambda z) \sin (2 \pi[r \cos (\theta+\Theta)+\Delta]) \cos (\theta+\Theta), \\
w & =-(2 \pi)^{-1} \lambda \exp (\lambda z) \sin (2 \pi[r \cos (\theta+\Theta)+\Delta]) \sin (\theta+\Theta), \\
p & =(1-\exp \lambda z) / 2 .
\end{aligned}
$$

As shown in Fig. 3, the displacement $\Delta$ ensures that flow crosses the cylindricalcoordinate axis, which with $\Theta$ a non-rational multiple of $\pi$, ensures that all the axial terms in the Navier-Stokes equations are exercised, for real and imaginary parts of all modes.

Two spectral element meshes for the meridional semi-plane are used in convergence testing of the formulation and are shown in Fig. 4; the internal mesh nodes are illustrated for tensor-product interpolants which with polynomial order $N_{\mathrm{p}}=11 \mathrm{in}$ each direction. The element geometries for the mesh in Fig. 4 (b) were chosen to ensure that some elements are non-orthogonal, and in particular, are not orthogonal to the axis, a factor that was shown to be an issue for a different formulation [7].

When computing the convergence tests, a constant number of Fourier modes, $N_{k}=24$, was maintained in the azimuthal direction: as we show below, this number was chosen to ensure that the errors were dominated by the spectral 

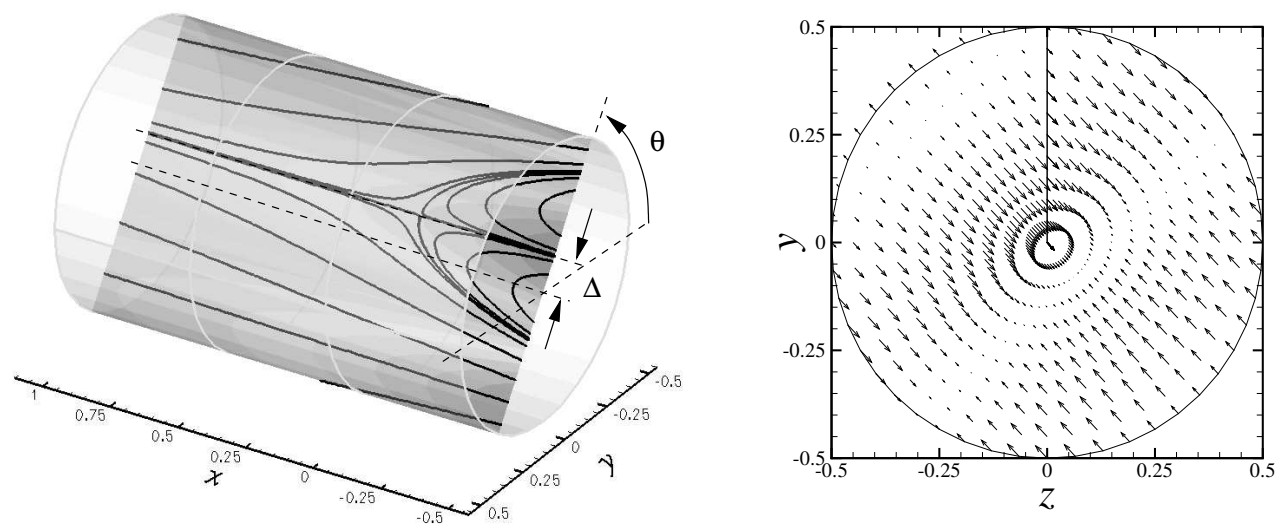

Fig. 3. With offset $\Delta \neq 0$, Kovasznay flow crosses the cylindrical coordinate axis, while an angular tilt $\Theta$ can be used to ensure that both the real and imaginary parts of all modes in the cylindrical formulation are exercised. The effect of these transformations is illustrated in this examination of streamlines and the Cartesian $(y$, $z)$-projection of velocity vectors at $x=z_{\mathrm{cyl}}=-0.25$, when $\Delta=0.1$ and $\Theta=0.75$.

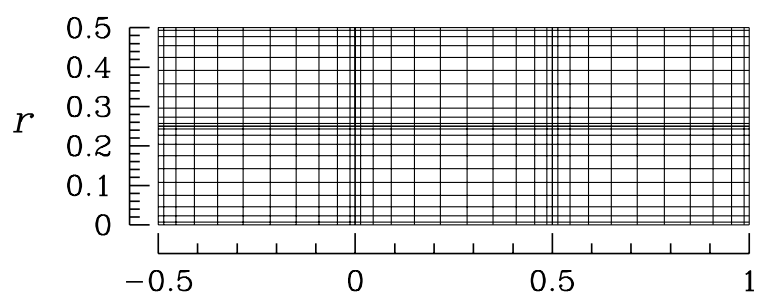

(a)

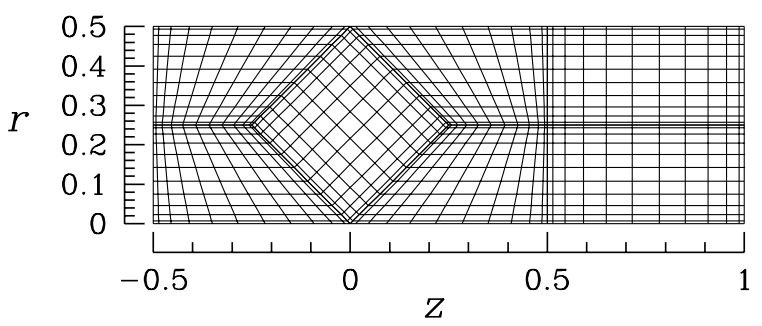

(b)

Fig. 4. Spectral element meshes used in the meridional semi-plane for convergence testing (shown at $N_{\mathrm{p}}=11$ ).

element discretisation in the meridional semi-plane. The Reynolds number $R e=40$ for all tests. At each interpolation order, $N_{\mathrm{p}}$, the exact velocity field was supplied as initial condition, and also as Dirichlet boundary condition on all exterior (non-axial) domain nodes. This initial value problem was then integrated forwards in time to steady state, after which the pointwise maximum deviation from the exact solution was found for each velocity component (i.e. $\left\|\boldsymbol{u}-\boldsymbol{u}_{\text {exact }}\right\|_{\infty}$, on a component-by-component basis). As the tests here were designed to examine the spatial convergence of a steady solution, firstorder (backwards-Euler, $J=1$ ) time-integration was employed. At the small timestep, $\Delta t=0.0025$, employed during testing (chosen for the CFL-stability limit on the finest mesh), the results were almost independent of timestep.

The outcomes of the tests, for the two meshes in Fig. 4, are illustrated for 


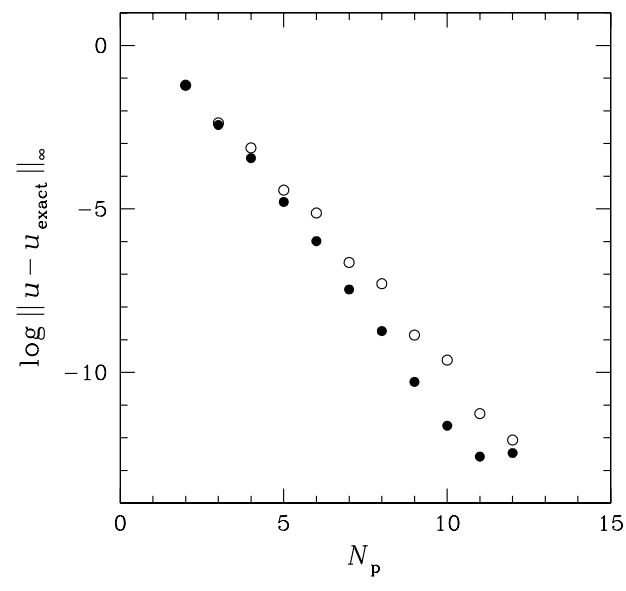

(a)

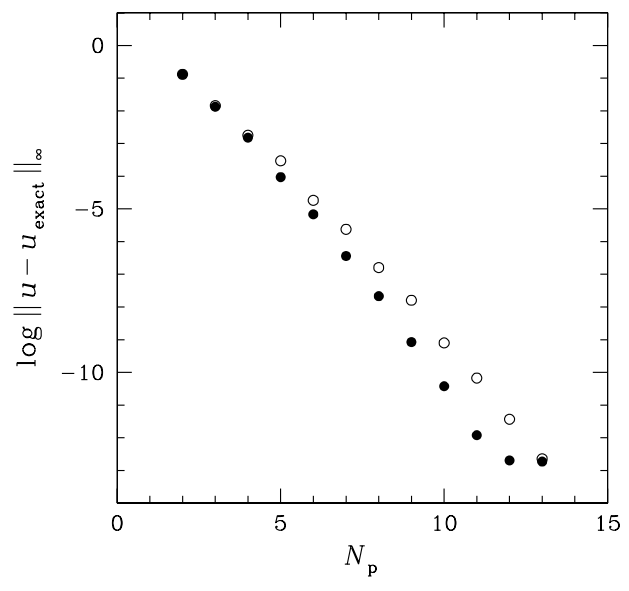

(b)

Fig. 5. Results of convergence testing on the meshes shown in Fig. 4, with $\Theta=0.75$ and $\Delta=0.1$, number of azimuthal Fourier modes $N_{k}=24 . N_{\mathrm{p}}$ is the order of spectral element Lagrange polynomial interpolants. Non-conservative formulation of nonlinear terms, $\bullet$; skew-symmetric form, ○.

the $u$ (axial) velocity component in Fig. 5 - results for the other two velocity components are very similar. It is clear that in both cases, a very good approximation to exponential convergence is achieved, until the limit of accuracy in double-precision calculations is approached $\left[\log \left(u-u_{\text {exact }}\right) \sim-13\right]$. An interesting point is that the non-conservative formulation of the nonlinear terms provides a faster convergence rate than the skew-symmetric form, in agreement with Cartesian-coordinate computations for this flow [20].

To demonstrate that azimuthal convergence is also exponential (as should be the case for Fourier expansions), we show in Fig. 6 the maximum error in $u$ as the number of Fourier modes $N_{k}$ is increased, for fixed $N_{\mathrm{p}}=11$. Exponential convergence sets in at $N_{k} \approx 8$, and saturates (in these double precision calculations) at $N_{k} \approx 18$. All test results presented here were computed with de-aliasing implemented in the azimuth direction, using the $3 / 2$ rule, however, as expected, the asymptotic convergence rate was not signficantly affected if de-aliasing was not employed.

\section{Discussion and conclusions}

In summary, the key formulation steps that we have adopted in our solution strategy for the Fourier-transformed Navier-Stokes equations in cylindrical coordinates are

(1) diagonalisation, to decouple the linear viscous terms using the transformation suggested by Orzsag \& Patera [19] (see $\S 2.1$ ); 


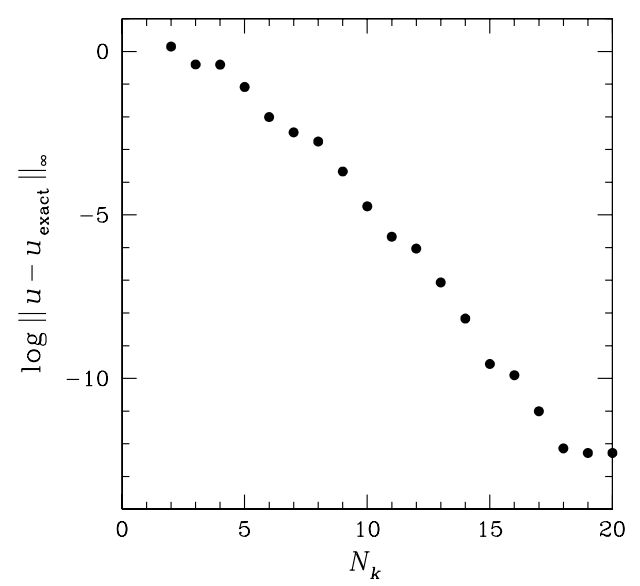

Fig. 6. Results of azimuthal (Fourier) convergence testing on the mesh of Fig. 4 (a), with $\Theta=0.75$ and $\Delta=0.1, N_{\mathrm{p}}=11 . N_{k}$ is the number of azimuthal Fourier modes. These computations employed the non-conservative formulation of the non-linear terms.

(2) symmetrisation, by multiplying the governing equation set by $r$ (see $\S 2.2$ );

(3) applying a Galerkin projection onto a discrete basis with a boundaryinterior decomposition, such as finite element, spectral element and $p$ type element discretisations (

These formulation steps can be applied regardless of the time discretisation employed. Further, for the primitive equations and the pressure Poisson equation all the geometric singularities at the axis of the cylindrical coordinate system do not contribute to the integral formulation. This last observation is a direct consequence of the integrands of the inner products that involve singular terms having numerators that go to zero faster than the denominators. From an implementation point of view this conveniently implies that all singular terms at the axis can be zeroed without loss of generality.

A variation on the approach we have described here would be to discretely multiply all quadrature weights by appropriate values of $r$. As demonstrated in App. A this manipulation is analogous to using a Gauss-Radau-Jacobi $(0,1)$ quadrature rule. From an implementation standpoint this treatment is superficially attractive since no further evaluation of the radial term is required when evaluating integrals. All geometrically singular terms in the primitive equations (and all quadratures on the left-hand-sides of the Galerkin projections for either the Poisson or Helmholtz operators) are not affected by this manipulation. However, problems can arise if a velocity (or pressure) correction scheme is employed in which a pressure Poisson equation must be solved. Following the discussion in $\S 3.4$, if the symmetrisation factor $r$ is absorbed into the quadrature weights when forming the right-hand-side of the pressure Poisson equation, then the integrand for the $k=0$ Fourier mode will now be singular at the axis. On the left-hand-side there would be no problem in 
this particular case because the mode-constant $\sigma^{2}=k^{2}=0$. If the singular integrand on the right-hand-side is arbitrarily set to zero at $r=0$, then we have incomplete quadrature of a singular integral, which, as shown in App. A, reduces the convergence rate from exponential to algebraic. The variation just outlined was adopted in the numerical method used in the non-axisymmetric simulations of [4] (and the reduced convergence rate was noted therein), although for that problem the reduced convergence was not an issue, as flow at the axis retained axisymmetry. It is interesting that the reduced convergence arises through nonlinear interactions, with the $k=1$ cross-axial flow contaminating the pressure solution for the axisymmetric mode. Thus, it would not compromise the accuracy of either a Stokes solution, an axisymmetric Navier-Stokes solution, or a linear stability analysis.

To properly evaluate the cylindrical coordinate implementation it is therefore necessary to have a fully three-dimensional, axis-crossing validation problem to exercise all terms in the Navier-Stokes equations. As we have shown in $\S 4$, such a problem can be constructed by considering the offset Kovasznay problem. To our knowledge no other paper has demonstrated the spectral convergence of a three-dimensional non-axisymmetric test case.

A popular alternative to velocity (or pressure) correction schemes is to directly invert the Stokes operator which is a mixed-type problem and typically requires a $\mathbb{P}_{N}-\mathbb{P}_{N-2}$ discretisation for velocity and pressure, respectively $[6,7]$. In these methods, the decoupling of the pressure and velocity is done at the discrete level and so no pressure boundary conditions are required. In the case of the cylindrical coordinate system which has been symmetrised by a factor of $r$ there can be a degeneracy in the pressure of the global system, as discussed by Gerritsma \& Phillips [7], if Gauss-Lobatto-Legendre quadrature is employed in elements that touch the axis. In the work of [7] the issue was addressed by using a spectral element discretisation and quadrature rule with nodal points in the radial direction based on the zeros of the $(0,1)$ Jacobi polynomials and absorbing the factor of $r$ into the quadrature weights (see App. A for definition of $(0,1)$ quadrature rules).

The use of different quadrature rules can also be considered in the context of the velocity correction schemes employed in this paper. Indeed in $[7,8,23]$, within elements that were adjacent to the axis, radial Lagrange nodal interpolants with nodes based in Gauss-Lobatto-Jacobi quadrature were employed. More specifically, the internal nodes of the special bases were related to zeros of $(0,1)$ Jacobi polynomials. An advantage of the Gauss-Lobatto-Jacobi $(0,1)$ quadrature is that a scaled radial weight function is directly absorbed into the quadrature weights while maintaining the accuracy of the integration: this should provide a slightly higher asymptotic convergence rate, since the polynomial order of the integrand is lowered. This is in contrast to discretely multiplying the Gauss-Lobatto-Legendre weights by the radius, where the accuracy 
of the integration is reduced by one order. In the Gauss-Lobatto-Jacobi $(0,1)$ approach, all the indeterminate forms on the left-hand-sides of equations (37) and (50-52) can be evaluated through l'Hopital's rule and at the same time the symmetry of the system of equations is retained. As we have demonstrated however, it is not necessary to explicitly evaluate these terms if one uses the standard Gauss-Lobatto-Legendre nodal expansions. We also note that in hierarchical spectral/hp-type element methods [14], the quadrature order is typically defined independently of the expansion polynomial order, and so the integrand can be evaluated consistently, without potential loss of accuracy.

A subtle feature of choosing a Gauss-Lobatto-Jacobi $(0,1)$ quadrature rule emerges if solution of a pressure Poisson equation is required. All the terms on the left-hand-side of the equation can be correctly evaluated, but again, in order to avoid a singular integral on the right-hand-side terms, the radius must not be incorporated into the quadrature weights, but directly distributed into the right-hand-side divergence operator. The implication in the spectral element setting is that the quadratures on the right-hand-side of the pressure Poisson equation should always be performed using Gauss-Lobatto-Legendre quadrature, after the integrands have been projected (if necessary) onto the Gauss-Lobatto-Legendre mesh points, rather than through Gauss-LobattoJacobi $(0,1)$ quadrature.

\section{Acknowledgements}

We would like to thank D. J. Newman for his help and discussions in the early stages of this work, and also acknowledge input from A. G. Tomboulides and D. M. McIver. This work was partially supported through the Merit Allocation Scheme of the Australian Partnership for Advanced Computing. The second author would like to acknowledge financial support from the Royal Academy of Engineering in the form of a Global Research Award.

\section{A Incomplete quadrature evaluation of the integral $\int_{0}^{R}[g(r) / r] r \mathrm{~d} r$ when $\lim _{r \rightarrow 0} g(r) \neq 0$.}

In our discussion of the treatment of geometric singularities that arise in the cylindrical coordinates description of the pressure Poisson equation we are interested in treating integrals of the form

$$
I=\int_{0}^{R}\left(\frac{g(r)}{r}\right) r \mathrm{~d} r=\int_{-1}^{1}\left(\frac{g(\xi)}{1+\xi}\right) \frac{R}{2}(1+\xi) \mathrm{d} \xi
$$


where $r(\xi)=(1+\xi) R / 2$ and $\lim _{r \rightarrow 0} g(r) \neq 0$. This can occur in the $\partial_{r} r \hat{v}_{0}^{*}$ term on the right-hand-side of (37), if $r$ is subsumed into the quadrature weights, which then generates an integral which has the one-dimensional equivalent of type

$$
\int_{0}^{R}\left(\frac{\hat{v}_{0}^{*}}{r}\right) r \mathrm{~d} r
$$

where $\left.\hat{v}_{0}^{*}\right|_{r=0} \neq 0$, in general (see $\S 3.4$ ). From inspection of equation (A.1) the most straightforward way of handling this integral is through analytically cancelling the factors of $r$ and directly integrating $g(r)$. However in a numerical implementation, the construction of terms such as $g(r) / r$ may be performed independently of the integration with respect to $r$ over the interval $[-1,1]$. To understand the consequence of separating the integrand into $g(r) / r$ multiplied by $r$ we first need to review some details of Gaussian quadrature.

Consider the function $g(\xi)$ approximated by a Lagrange polynomial, $h_{q}(\xi)$ defined through a set of points $\xi_{q}$ for $0 \leq q \leq Q$, i.e.

$$
g_{1}(\xi)=\sum_{q=0}^{Q} g\left(\xi_{q}\right) h_{q}(\xi)+\epsilon_{h}(\xi)
$$

where $\epsilon_{h}(\xi)$ is the approximation error. We can derive a general quadrature rule by integrating equation (A.2) over the interval $\xi \in[-1,1]$ with respect to the weighting function $(1-\xi)^{\alpha}(1+\xi)^{\beta}$ to obtain

$$
\int_{-1}^{1}(1-\xi)^{\alpha}(1+\xi)^{\beta} g(\xi) \mathrm{d} \xi=\sum_{q=0}^{Q} g_{1}\left(\xi_{q}^{\alpha, \beta}\right) w_{q}^{\alpha, \beta}+\epsilon_{I}
$$

where

$$
w_{q}^{\alpha, \beta}=\int_{-1}^{1}(1-\xi)^{\alpha}(1+\xi)^{\beta} h_{q}(\xi) \mathrm{d} \xi, \quad \epsilon_{I}=\int_{-1}^{1} \epsilon_{h}(\xi) \mathrm{d} \xi
$$

The Gauss-Lobatto-Legendre quadrature rule

$$
\int_{-1}^{1} g(\xi) \mathrm{d} \xi=\sum_{q=0}^{Q} g_{1}\left(\xi_{q}^{0,0}\right) w_{q}^{0,0}+\epsilon_{I}
$$

is defined by the choice of the Legendre weights, $\alpha=0, \beta=0$ and defining the zeros, $\xi_{q}^{0,0}$, to be $Q+1$ roots of the polynomial $(1-\xi)(1+\xi) P_{Q-1}^{1,1}(\xi)$ where $P_{Q-1}^{1,1}(\xi)$ is the $(1,1)$ Jacobi polynomial of degree $Q-1$. The choice of the zeros is pertinent since this makes the integration exact for integrands which are in the polynomial space $\mathbb{P}_{2 Q-1}[9,14]$. 
The Gauss-Radau-Jacobi(0,1) quadrature rule

$$
\int_{-1}^{1}(1+\xi) g(\xi) \mathrm{d} \xi=\sum_{q=1}^{Q} g_{1}\left(\xi_{q}^{0,1}\right) w_{q}^{0,1}+\epsilon_{I}
$$

(note that the summation starts from $q=1$ ) is defined by choosing the weights $\alpha=0, \beta=1$ and defining the zeros, $\xi_{q}^{0,1}$ to be the roots of the polynomial $(1+\xi) P_{Q-1}^{1,1}(\xi)$. This rule contains a zero only at end point $\xi=1$ and is exact for integrands which are in the polynomial space $\mathbb{P}_{2 Q-2}$.

From the definition above the zeros of the two rules are the same for $1 \leq q \leq Q$, i.e.

$$
\xi_{q}^{0,0}=\xi_{q}^{0,1} \quad \text { for } \quad q=1, \ldots, Q
$$

To determine the connection between the weights $w_{q}^{0,0}$ and $w_{q}^{0,1}$ we first note that for $1 \leq q \leq Q$ they are defined in terms of the Lagrange polynomials as

$$
w_{q}^{0,0}=\int_{-1}^{1} h_{q}^{0,0}(\xi) \mathrm{d} \xi, \quad w_{q}^{0,1}=\int_{-1}^{1}(1+\xi) h_{q}^{0,1}(\xi) \mathrm{d} \xi
$$

where the superscript Lagrange polynomials can be defined as

$$
\begin{aligned}
& h_{q}^{0,0}(\xi)=\frac{(1-\xi)(1+\xi) P_{Q}^{1,1}(\xi)}{\left[(1-\xi)(1+\xi) P_{Q}^{1,1}(\xi)\right]_{x=x_{q}}^{\prime}\left(\xi-\xi_{q}\right)}, \\
& h_{q}^{0,1}(\xi)=\frac{(1-\xi) P_{Q}^{1,1}(\xi)}{\left[(1-\xi) P_{Q}^{1,1}(\xi)\right]_{x=x_{q}}^{\prime}\left(\xi-\xi_{q}\right)} .
\end{aligned}
$$

Insertion of (A.8) into the definitions (A.7) and comparison leads us to the fact that $w_{q}^{0,0}=w_{q}^{0,1} /\left(1+\xi_{q}\right)$.

Returning to the issue of evaluating integral (A.1) by splitting the evaluation of the integrand into $g(r) / r$ multiplied by $r$. In this case if $g(r) / r$ is evaluated at $r=0$ (owing to the presence of a quadrature point at this location) then we have to determine an appropriate value for our implementation. Had $\lim _{r \rightarrow 0} g(r) \rightarrow 0$, then setting $g(r) / r=0$ would not have caused a error in the integral (A.1), as this assumption would have been consistent with the multiplication by $r$ in the numerator of the integrand. This situation arises for most of the geometric singular terms in the cylindrical coordinate system. Nevertheless even in the case where $\lim _{r \rightarrow 0} g(r) \neq 0$ it is possible to demonstrate that setting $g(r) /\left.r\right|_{r=0}=0$ still leads to a consistent approximation of the integral $I$, although such an implementation destroys the exponential convergence rate of approximation.

To demonstrate this, we start with the Gauss-Lobatto-Legendre representa- 

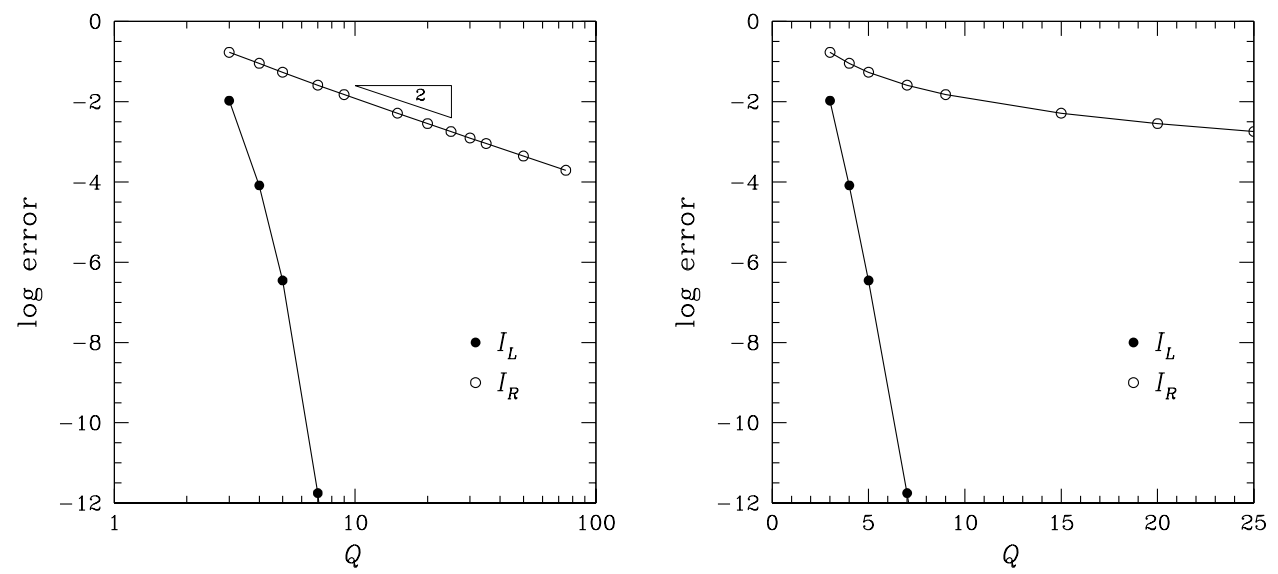

Fig. A.1. Error in numerical approximation to the integral $\int_{-1}^{1}(1+\xi) \cos (\xi) /(1+\xi) \mathrm{d} \xi$ using Gauss-Lobatto-Legendre quadrature, $I_{L}$ and Gauss-Radau-Jacobi $(0,1), I_{R}$ quadrature on semi-log and log-log axis.

tion of integral (A.1), which is

$$
I_{L}=\sum_{q=0}^{Q}\left(1+\xi_{q}^{0,0}\right)\left(\frac{g\left(\xi_{q}^{0,0}\right)}{1+\xi_{q}^{0,0}}\right) \frac{R}{2} w_{q}^{0,0} .
$$

If $g\left(\xi_{0}^{0,0}\right) /\left(1+\xi_{0}^{0,0}\right)=0$, we can write expression (A.9) using equations (A.6) and (A.7) as

$$
I_{R}=\sum_{q=1}^{Q}\left(\frac{g\left(\xi_{q}^{0,1}\right)}{1+\xi_{q}^{0,1}}\right) \frac{R}{2} w_{q}^{0,1}
$$

So although enforcing the condition that $g\left(\xi_{0}^{0,0}\right) /\left(1+\xi_{0}^{0,0}\right)=0$ would initially appear to make equation (A.9) an inconsistent approximation to the integral (A.1) we observe from equation (A.10) that zeroing this term is equivalent to approximating the integral (A.1) with a consistent Gauss-Radau-Jacobi $(0,1)$ quadrature rule. Nevertheless the continuous integrand of the Gauss-RadauJacobi $(0,1)$ rule is the function $g(\xi) /(1+\xi)$ which is singular at $\xi=-1$ and so the numerical approximation of this integral will not demonstrate exponential convergence as a function of quadrature order $Q$. This point is illustrated in Fig. A.1 where we show the error in evaluating the integral $\int_{-1}^{1}(1+\xi)[\cos (\xi) /(1+\xi)]$ d $\xi[$ i.e. $g(\xi)=\cos (\xi)]$ using Gauss-Lobatto-Legendre quadrature as in equation (A.9) and Gauss-Radau-Jacobi $(0,1)$ quadrature as in equation (A.10). For this case we see that the convergence rate using $I_{L}$ is exponential in quadrature order $Q$ whereas when evaluating $I_{R}$ the convergence rate is only algebraic and is of $O\left(Q^{2}\right)$. 


\section{References}

[1] C. H. Amon. Spectral element-Fourier method for transitional flows in complex geometries. AIAA J., 31(1):42-48, 1993.

[2] C. H. Amon and A. T. Patera. Numerical calculation of stable three-dimensional tertiary states in grooved-channel flow. Phys. Fluids A, 1(2):2005-2009, 1989.

[3] G. K. Batchelor and A. E. Gill. Analysis of the stability of axisymmetric jets. J. Fluid Mech., 14:529-551, 1962.

[4] H. M. Blackburn and J. M. Lopez. Modulated rotating waves in an enclosed swirling flow. J. Fluid Mech., 465:33-58, 2002.

[5] C. Canuto, M. Y. Hussaini, A. Quarteroni, and T. A. Zang. Spectral Methods in Fluid Dynamics. Springer, 1988. 2nd printing.

[6] M. O. Deville, P. F. Fischer, and E. H. Mund. High-Order Methods for Incompressible Fluid Flow. Cambridge University Press, 2002.

[7] M. I. Gerritsma and T. N. Phillips. Spectral element methods for axisymmetric Stokes problems. J. Comput. Phys., 164:81-103, 2000.

[8] B. Ghidersa and J. Dǔsek. Breaking of axisymmetry and onset of unsteadiness in the wake of a sphere. J. Fluid Mech., 423:33-69, 2000.

[9] A. Ghizzetti and A. Ossicini. Quadrature Formulae. Academic Press, 1970.

[10] J. L. Guermond and J. Shen. Velocity-correction projection methods for incompressible flows. SIAM J. Numer. Anal., 41(1):112-134, 2003.

[11] T. J. R. Hughes. The Finite Element Method: Linear Static and Dynamic Finite Element Analysis. Prentice-Hall, 1987.

[12] G. E. Karniadakis. Spectral element-Fourier methods for incompressible turbulent flows. Comp. Meth. Appl. Mech. E Engng, 80:367-380, 1990.

[13] G. E. Karniadakis, M. Israeli, and S. A. Orszag. High-order splitting methods for the incompressible Navier-Stokes equations. J. Comput. Phys., 97(2):414$443,1991$.

[14] G. E. Karniadakis and S. J. Sherwin. Spectral/hp Element Methods for CFD. Oxford University Press, 1999.

[15] L. I. G. Kovasznay. Laminar flow behind a two-dimensional grid. Proc. Cambridge Phil. Soc., page 44, 1948.

[16] A. G. Kravchenko, P. Moin, and K. Shariff. B-spline method and zonal grids for simulations of complex turbulent flows. J. Comput. Phys., 151:757-789, 1999.

[17] J. M. Lopez, F. Marques, and J. Shen. An efficient spectral-projection method for the Navier-Stokes equation in cylindrical geometries II. Three dimensional cases. J. Comput. Phys., 176:384-401, 2002. 
[18] J. T. Oden, W. Wu, and V. Legat. An $h p$ adaptive strategy for the finite element approximations of the Navier-Stokes equations. Intnl J. Num. Meth. Fluids, 20:831-851, 1995.

[19] S. A. Orszag and A. T. Patera. Secondary instability of wall-bounded shear flows. J. Fluid Mech., 128:347-385, 1983.

[20] E. M. Rønquist. Convection treatment using spectral elements of different order. Intnl J. Num. Meth. Fluids, 22:241-264, 1996.

[21] J. Shen. Efficient spectral-Galerkin methods III: Polar and cylindrical geometries. SIAM J. Sci. Comput., 18(6):1583-1604, 1997.

[22] G. Strang and G. J. Fix. An Analysis of the Finite Element Method. PrenticeHall, 1973.

[23] A. G. Tomboulides and S. A. Orszag. Numerical investigation of transitional and weak turbulent flow past a sphere. J. Fluid Mech., 416:45-73, 2000.

[24] L. S. Tuckerman. Divergence-free velocity fields in nonperiodic geometries. J. Comput. Phys., 80:403-411, 1989. 